Pacific Journal of Mathematics

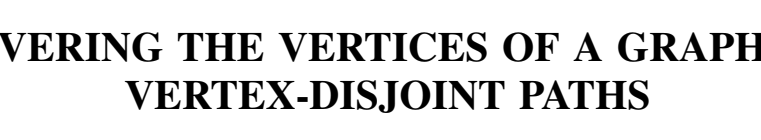




\section{COVERING THE VERTICES OF A GRAPH BY VERTEX-DISJOINT PATHS}

\section{Shahbaz NoORVASH}

Define the path-covering number $\mu(G)$ of a finite graph $G$ to be the minimum number of vertex-disjoint paths required to cover the vertices of $G$. Let $g(n, k)$ be the minimum integer so that every graph, $G$, with $n$ vertices and at least $g(n, k)$ edges has $\mu(G) \leqq k$. A relationship between $\mu(G)$ and the degree sequence for a graph $G$ is found; this is used to show that

$$
\frac{1}{2}(n-k)(n-k-1)+1 \leqq g(n, k) \leqq \frac{1}{2}(n-1)(n-k-1)+1
$$

A further extremal problem is solved.

1. Introduction. A graph $G$ is a finite collection $\mathscr{V}(G)$ of vertices (or points) some pairs of which are joined by a single edge; the collection of edges is denoted by $\mathscr{E}(G)$. H is a subgraph of $G$ if $\mathscr{V}(H) \subseteq \mathscr{V}(G)$ and $\mathscr{E}(H) \subseteq \mathscr{E}(G)$. If $H$ and $K$ are two vertex-disjoint graphs, $H \cup K$ is the graph with $\mathscr{V}(H \cup K)=\mathscr{V}(H) \cup \mathscr{V}(K)$ and $\mathscr{E}(H \cup K)=\mathscr{E}(H) \cup \mathscr{E}(K) ; \quad H+K$ is $H \cup K$ together with all $|\mathscr{V}(H)||\mathscr{V}(K)|$ possible choices of edges joining a vertex of $H$ to a vertex of $K$. $\bar{G}$ denotes the complement of $G ; \Gamma_{n}$ denotes the complete graph with $n$ vertices and $\Gamma_{m, n}$ denotes the complete bipartite graph, $\bar{\Gamma}_{m}+\bar{\Gamma}_{n}$.

Let $G$ be a graph. A path of length $n$ in $G$ is an ordered sequence $P=\left\langle a_{1}, a_{2}, \cdots, a_{n}\right\rangle$ of distinct points, where if $n \geqq 2, a_{i}$ is adjacent to $a_{i+1}$ for $1 \leqq i \leqq n-1 .\left\langle a_{1}, a_{2}, \cdots, a_{n}\right\rangle$ is the same path as $\left\langle a_{n}, a_{n-1}, \cdots, a_{1}\right\rangle$. If $P$ and $Q$ are paths, by $P * Q$ we shall mean that one end-point, $a$ of $P$, is adjacent to one end-point, $b$ of $Q$, and that $P * Q$ is formed by joining $a$ to $b$. More specifically we may write $P a * b Q$ or $P * b Q$ or $P a * Q$ to specify, in varying degrees, which end-point of $P$ is joined to which end-point of $Q$. Also, $\left\langle a_{1}, a_{2}, \cdots, a_{n}\right\rangle *\left\langle b_{1}, b_{2}, \cdots, b_{m}\right\rangle=$ $\left\langle a_{1}, a_{2}, \cdots, a_{n}, b_{1}, b_{2}, \cdots, b_{m}\right\rangle$ where $a_{n}$ must be adjacent to $b_{1}$. A Hamilton-path is a path of length $|\mathscr{V}(G)|$. A path-cover of $G$ is a collection, $\mathscr{S}$, of vertex-disjoint paths such that every vertex of $G$ lies on some path in $\mathscr{S}$. The path-covering number, denoted by $\mu(G)$, of $G$ is defined by:

$$
\mu(G)=\operatorname{Min}\{|\mathscr{S}|: \mathscr{S} \text { is a path-cover of } G\}
$$


A minimal path-cover (M.P.C.) of $G$ is a path-cover, $\mathscr{S}$ of $G$, with $|\mathscr{S}|=\mu(G)$.

We note that $\mu(G)$ is an invariant of $G$ and remark that a graph, $G$, has a Hamilton-path if and only if $\mu(G)=1$. It has been shown by Nash-Williams [1] and others that the problem of classifying all Hamiltonian graphs is equivalent to that of classifying all graphs which have a Hamilton-path. Thus a classification of all graphs with $\mu(G)=k$ $(k=1,2,3, \cdots)$ would also solve the Hamiltonian problem as a special case.

Historically, O, Ore [3] first introduced the graphical invariant $\mu$. In [2] some elementary properties of $\mu$ are derived. In $\$ 2$ we generalize a result of $O$. Ore (Theorem 2.1 in [3]) and in $\$ 3$ we consider two extremal problems involving $\mu$.

2. Valency considerations. In this section we derive a connection between the path-covering number and the degree sequence of a graph. We begin with some definitions:

Definition 2.1. Let $A$ be a finite set and $f$ a real-valued function defined on the collection of subsets of $A$. For $B \subseteq A$ and for any integer $i$ with $1 \leqq i \leqq|B|$, define the function $S_{i}$ by:

$$
S_{i}(f, B)=\sum_{\substack{C \subset B \\|C|=i}} f(C)
$$

Definition 2.2. If $G$ is a graph, $B \subseteq \mathscr{V}(G)$, and either $H \subseteq \mathscr{V}(G)$ or $H$ is a subgraph of $G$, then define the generalized valence function, $\rho$, by

$$
\begin{aligned}
\rho_{H}(B)= & \text { the number of vertices of } H \text { which are adjacent } \\
& \text { to every member of } B .
\end{aligned}
$$

If $x$ is a vertex of $G$, then we write $\rho(x)$ for $\rho_{G}(\{x\})$.

Definition 2.3. Let $G$ be a graph and $X \subseteq \mathscr{V}(G)$ with $|X|=k \geqq 2$. Define:

$$
D(G, X)=\frac{1}{k} S_{1}\left(\rho_{G}, X\right)-\sum_{i=1}^{k}(-1)^{i}\left(\frac{k-i}{k}\right) S_{i}\left(\rho_{G}, X\right) .
$$

The following lemma is easily verified: 
Lemma 2.4. If $X=\left\{x_{1}, x_{2}, \cdots, x_{k}\right\}$, and $1 \leqq m \leqq k-1$, then

$$
\sum_{i=1}^{k} S_{m}\left(f, X-\left\{x_{i}\right\}\right)=(k-m) S_{m}(f, X)
$$

We now state the main result of this section:

THEOREM 2.5. Let $G$ be a graph with $\mu=\mu(G) \geqq 2,|\mathscr{V}(G)|=n$ and $k$ an integer with $2 \leqq k \leqq \mu$, then there exists a set $X$ consisting of $k$ mutually non-adjacent vertices of $G$, satisfying:

$$
\mu \leqq n-D(G, X)
$$

Note that the case $k=2$ reduces to the result of Ore (Theorem 2.1 in [3]):

$$
\mu \leqq n-\rho\left(x_{1}\right)-\rho\left(x_{2}\right)
$$

Proof. Let $\mathscr{S}=\left\{P_{1}, P_{2}, \cdots, P_{\mu}\right\}$ be a M.P.C. for $G$. For each $1 \leqq i \leqq k$, let $x_{i}$ be an end-vertex of $P_{i}$. Since $\mathscr{S}$ is a M.P.C., $x_{i}$ is not adjacent to $x_{j}$ for $i \neq j$.

Let $X=\left\{x_{1}, x_{2}, \cdots, x_{k}\right\}$. We first show that for $1 \leqq i \leqq k$ and $1 \leqq j \leqq \mu$, the inequality:

$$
\rho_{P_{j}}\left(\left\{x_{i}\right\}\right) \leqq\left|P_{j}\right|-\left(1-\sum_{i=1}^{k-1}(-1)^{l} S_{l}\left(\rho_{P_{i}}, X-\left\{x_{i}\right\}\right)\right)
$$

holds. Let $P_{j}$ be the path $\left\langle a_{1}, a_{2}, \cdots, a_{t}\right\rangle$, let $1 \leqq m \leqq k, m \neq i$, and consider the following cases:

(i) $i=j$. In this case assume that $x_{i}=a_{1}$.

(ii) $m=j$. In this case assume that $x_{m}=a_{t}$.

(iii) $m \neq j$ and $i \neq j$.

Let

$$
\begin{aligned}
A & =\left\{r: a_{r} \text { is adjacent to } x_{i}\right\}, \\
B_{m} & =\left\{r: a_{r-1} \text { is adjacent to } x_{m}\right\}
\end{aligned}
$$

and

$$
B=\bigcup_{\substack{1 \leqq m \leqq k \\ m \neq i}} B_{m}
$$

We claim that $A \cap B_{m}=\phi$, for if $r \in A \cap B_{m}$, then in each case we can 
construct a path-cover, $\mathscr{T}$ for $G$, as follows (see Figure 2.8):

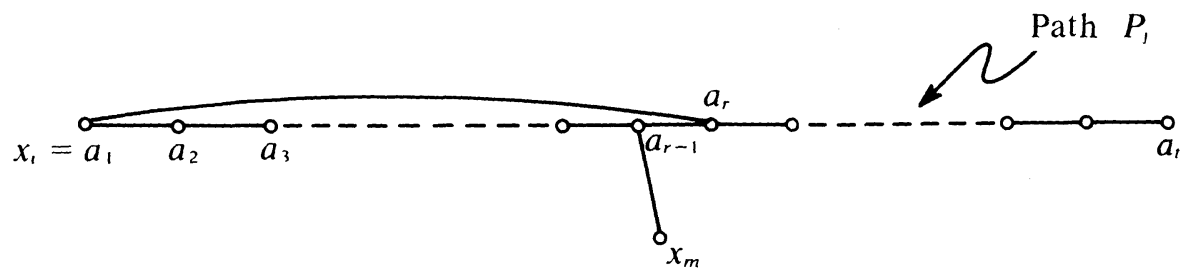

Case (i)

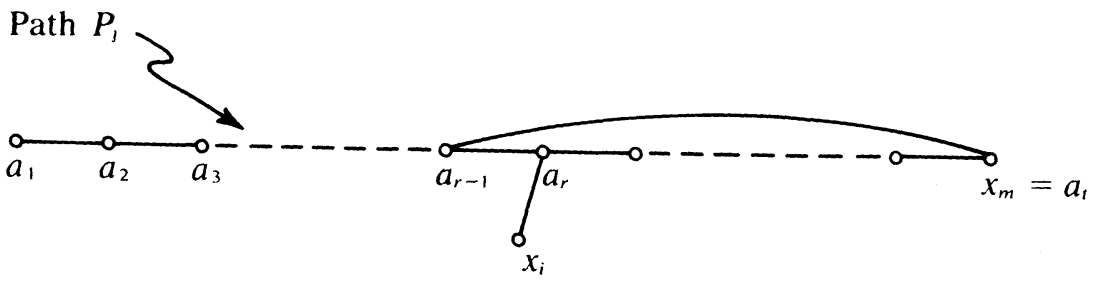

Case (ii)

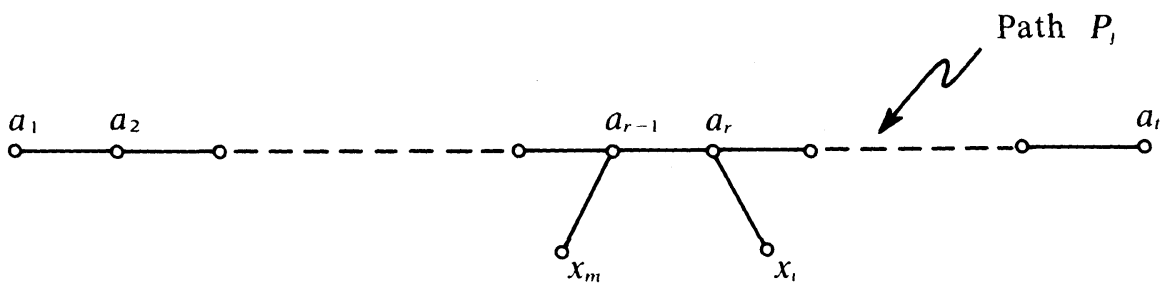

Case (iii)

Figure 2.8

In case (i), let:

$$
\mathscr{T}=\mathscr{S} \cup\left\{\left\langle a_{t}, a_{t-1}, \cdots, a_{r}, x_{i}, a_{2}, a_{3}, \cdots, a_{r-1}\right\rangle * x_{m} P_{m}\right\}-\left\{P_{i}, P_{m}\right\}
$$


In case (ii), let:

$$
\mathscr{T}=\mathscr{S} \cup\left\{\left\langle a_{1}, a_{2}, \cdots, a_{r-1}, x_{m}, a_{t-1}, a_{t-2}, \cdots, a_{r}\right\rangle * x_{i} P_{i}\right\}-\left\{P_{t}, P_{m}\right\}
$$

In case (iii), let:

$$
\mathscr{T}=\mathscr{S} \cup\left\{\left\langle a_{1}, \cdots, a_{r-1}\right\rangle * x_{m} P_{m},\left\langle a_{t}, a_{t-1}, \cdots, a_{r}\right\rangle * x_{i} P_{i}\right\}-\left\{P_{t}, P_{j}, P_{m}\right\}
$$

In either case, $|\mathscr{T}|=|\mathscr{S}|-1<|\mathscr{S}|$, contradicting the minimality of $\mathscr{S}$. Hence $A \cap B_{m}=\phi$. Also, in each case $a_{1} \notin A$; so $A \subseteq$ $P_{j}-B \cup\left\{a_{1}\right\}$. This gives $|A| \leqq\left|P_{j}\right|-\left|B \cup\left\{a_{1}\right\}\right|$, since $B \cup\left\{a_{1}\right\} \subseteq$ $P_{j}$. But then, since $a_{1} \notin B$, we get:

$$
|A| \leqq\left|P_{j}\right|-(1+|B|) \text {. }
$$

For $1 \leqq m \leqq k$, let:

$$
C_{m}=\left\{r: a_{r} \text { is adjacent to } x_{m}\right\}
$$

Then since $x_{m}$ is not adjacent to $a_{1},\left|C_{m}\right|=\left|B_{m}\right|$ and:

$$
\begin{aligned}
|B| & =\left|\bigcup_{\substack{1 \leq m \leq k \\
m \neq i}} B_{m}\right|=\left|\bigcup_{\substack{1 \leq m \leq k \\
m \neq i}} C_{m}\right| \\
& =\sum_{l=1}^{k-1}(-1)^{l+1} \sum_{\substack{1 \leqq m_{1}<m_{m}<\cdots<m_{l} \leqq k \\
m_{1}, m_{2}}, \cdots, m_{l} \neq i}\left|C_{m_{1}} \cap C_{m_{2}} \cap \cdots \cap C_{m_{l}}\right| \\
& =-\sum_{l=1}^{k-1}(-1)^{l} S_{l}\left(\rho_{P_{l}}, X \cdot-\left\{x_{i}\right\}\right) .
\end{aligned}
$$

So since $|A|=\rho_{P_{i}}\left(\left\{x_{i}\right\}\right),(2.7)$ follows from (2.9) and (2.10). Summing (2.7) for $1 \leqq i \leqq k$ and applying Lemma 2.4 , we get:

$$
S_{1}\left(\rho_{P_{i}}, X\right) \leqq k\left|P_{j}\right|-\left(k-\sum_{l=1}^{k-1}(-1)^{l}(k-l) S_{l}\left(\rho_{P_{1}}, X\right)\right) .
$$


Summing (2.11) for $1 \leqq j \leqq \mu$, we get:

$$
S_{1}\left(\rho_{G}, X\right) \leqq k n-\left(k \mu-\sum_{l=1}^{k-1}(-1)^{l}(k-l) S_{l}\left(\rho_{G}, X\right)\right) .
$$

from which (2.6) follows.

\section{Extremal problems.}

Definition 3.1. Let $k$ and $n$ be integers with $1 \leqq k \leqq n$. Define:

$$
\begin{gathered}
g(n, k)=\operatorname{Min}\{m: \text { every graph, } G, \text { with }|\mathscr{V}(G)|=n \text { and } \\
|\mathscr{E}(G)| \geqq m \text { has } \mu(G) \leqq k\} .
\end{gathered}
$$

In this section we determine bounds for $g(n, k)$. See [4] for techniques in proving the following:

LEMMA 3.2.

$$
\begin{aligned}
& \sum_{i=1}^{k=1}(-1)^{i}\left(\frac{k-i}{k}\right)\left(\begin{array}{l}
k \\
i
\end{array}\right)=-1 \quad \text { if } \quad k \geqq 2, \\
& \sum_{i=2}^{k}(-1)^{i}(k-i+1)\left(\begin{array}{c}
k \\
i-1
\end{array}\right)=k \quad \text { if } \quad k \geqq 2, \\
& \sum_{i=2}^{j}(-1)^{i}(k-i+1)\left(\begin{array}{c}
j-1 \\
i-1
\end{array}\right)=k \quad \text { if } \quad 3 \leqq \mathrm{j} \leqq \mathrm{k} .
\end{aligned}
$$

LEMMA 3.6. Let $K$ be a graph with $|\mathscr{V}(K)|=s \geqq 1$, and let $k$ be an integer with $k \geqq 2$, and suppose $H=\bar{\Gamma}_{k}+K$, then:

$$
D\left(H, \mathscr{V}\left(\bar{\Gamma}_{k}\right)\right)=2 s
$$

Proof. For $1 \leqq i \leqq k-1$ and $B \subseteq \mathscr{V}\left(\bar{\Gamma}_{k}\right)$ with $|B|=i$, each member of $B$ is adjacent to every member of $\mathscr{V}(K)$. There are $\left(\begin{array}{l}k \\ i\end{array}\right)$ choices for $B$ and $|\mathscr{V}(K)|=s$; thus:

$$
S_{i}\left(\rho_{H}, \mathscr{V}\left(\bar{\Gamma}_{k}\right)\right)=s\left(\begin{array}{l}
k \\
i
\end{array}\right)
$$


This gives:

$$
\begin{aligned}
D\left(H, \mathscr{V}\left(\bar{\Gamma}_{k}\right)\right. & =\frac{s}{k}\left(\begin{array}{c}
k \\
1
\end{array}\right)-\sum_{i=1}^{k-1}(-1)^{i} s\left(\frac{k-i}{k}\right)\left(\begin{array}{l}
k \\
i
\end{array}\right) \\
& =s\left[1-\sum_{i=1}^{k-1}(-1)^{i}\left(\frac{k-i}{k}\right)\left(\begin{array}{l}
k \\
i
\end{array}\right)\right] \\
& =2 s, \quad \text { using } \quad(3.3) .
\end{aligned}
$$

THEOREM 3.7. For $1 \leqq k \leqq n$,

$$
g(n, k) \leqq \frac{1}{2}(n-1)(n-k-1)+1 .
$$

Proof. Let $G$ be a graph with $|\mathscr{V}(G)|=n$, and $|\mathscr{E}(G)| \geqq$ $\frac{1}{2}(n-1)(n-k-1)+1$. Suppose $\mu(G)>k$ and $X=\left\{x_{1}, x_{2}, \cdots, x_{k}, x_{k+1}\right\}$ is a set of mutually nonadjacent vertices of $G$.

$G$ may be considered to have been obtained from the complete graph $\Gamma_{n}$ through the elimination of at most:

$$
\frac{1}{2} n(n-1)-\frac{1}{2}(n-1)(n-k-1)-1=\frac{1}{2}(n-1)(k+1)-1
$$

edges. $\frac{1}{2} k(k+1)$ are removed in obtaining, from $\Gamma_{n}$, the graph $H$ in which only members of $X$ are nonadjacent. Thus, to obtain $G$ from $H$, at most:

$$
\frac{1}{2}(n-1)(k+1)-1-\frac{1}{2} k(k+1)=\frac{1}{2}(n-k-1)(k+1)-1
$$

edges are removed from $H$.

We wish to compute $D(G, X)$. By Lemma 3.6,

$$
D(H, X)=2(n-k-1) .
$$

Now suppose that at some stage in the transformation from $H$ to $G$, we have obtained a graph $K$ with $\mathscr{E}(H) \supseteq \mathscr{E}(K) \supseteq \mathscr{E}(G)$ and $\mathscr{V}(K)=$ $\mathscr{V}(H)=\mathscr{V}(G)$. Let $L=K-e$ where $e \in \mathscr{E}(K)-\mathscr{E}(G)$. We wish to know the effect, $f(e)=D(L, X)-D(K, X)$, on $D$, of removing the edge $e$. Since $e$ is an edge of $H$, it cannot join two points of $X$. If neither end-point of $e$ is in $X$, then $f(e)=0$ since $S_{l}\left(\rho_{K}, X\right)=S_{i}\left(\rho_{L}, X\right)$ for $1 \leqq i \leqq k$. Now suppose that one end-point, $y_{1}$, of $e$ is in $X$ and that the other end-point, $v$, is not in $X$. Let $y_{1}, y_{2}, \cdots, y_{j}$ be the points of $X$ which are adjacent to $v$ in the graph $K$. Note that $1 \leqq j \leqq k+1$. 
If $1 \leqq i \leqq j$ and $B \subseteq\left\{y_{2}, y_{3}, \cdots, y_{j}\right\}$ with $|B|=i-1$, and $C=$ $B \cup\left\{y_{1}\right\}$, then $|C|=i$ and $v$ is adjacent to every member of $C$ in the graph $K$ but not in the graph $L$. There are $\left(\begin{array}{l}j-1 \\ i-1\end{array}\right)$ choices for such a set $C$. Furthermore, for any other combination of a vertex, $t$, and a set $A \subseteq X$ with $|A|=i, t$ is adjacent to every member of $A$ in the graph L. Thus:

$$
S_{i}\left(\rho_{L}, X\right)-S_{i}\left(\rho_{K}, X\right)=\left\{\begin{array}{ccr}
-\left(\frac{j-1}{i-1}\right) & \text { for } & i \leqq i \leqq j \\
0 & \text { for } & i>j
\end{array}\right.
$$

This gives:

$$
\begin{aligned}
f_{j} & = \begin{cases}-\left[\frac{1}{k+1}-\sum_{i=1}^{k}(-1)^{i}\left(\frac{k-i+1}{k+1}\right)\left(\begin{array}{c}
k \\
i-1
\end{array}\right)\right] & \text { if } j=k+1 \\
-\left[\frac{1}{k+1}-\sum_{i=1}^{j}(-1)^{i}\left(\frac{k-i+1}{k+1}\right)\left(\begin{array}{l}
j-1 \\
i=1
\end{array}\right)\right] & \text { if } 1 \leqq j \leqq k\end{cases} \\
& = \begin{cases}-\frac{1}{k+1}\left[k+1-\sum_{i=2}^{k}(-1)^{i}(k-i+1)\left(\begin{array}{c}
k \\
i-1
\end{array}\right)\right] & \text { if } j=k+1 \\
-\frac{1}{k+1}\left[k+1-\sum_{i=2}^{k}(-1)^{i}(k-i+1)\left(\begin{array}{l}
j-1 \\
i-1
\end{array}\right)\right] & \text { if } 2 \leqq j \leqq k \\
-1 & \text { if } j=1\end{cases} \\
& =\left\{\begin{array}{lll}
-\frac{1}{k+1} & \text { if } 3 \leqq j \leqq k+1 \\
-\frac{2}{k+1} & \text { if } j=2 \\
-1 & \text { if } j=1
\end{array}\right.
\end{aligned}
$$

using (3.4) and (3.5).

Notice that $f_{1} \leqq f_{2} \leqq \cdots \leqq f_{k} \leqq f_{k+1}<0$ and that in order to realize the effect $f_{j}$, edges with effects $f_{k+1}, f_{k}, \cdots, f_{j+1}$ must first be removed. Hence when $(k+1)$ edges are removed, the combined effect is at least:

$$
\sum_{i=1}^{k+1} f_{i}=-2
$$


So if $r$ edges are removed in obtaining $G$ from $H$,

$$
D(G, X)-D(H, X) \geqq-\frac{2 r}{k+1}
$$

Using (3.9) and (3.10) in (3.11) now gives:

$$
D(G, X) \geqq[2(n-k-1)-(n-k-1)+2 /(k+1)]>n-k-1 .
$$

But Theorem 2.5 guarantees the existence of a set $X$ as constructed above, and satisfying:

$$
D(G, X) \leqq n-\mu(G) \leqq n-k-1 .
$$

This contradicts (3.12) and completes the proof of the theorem.

COROllary 3.13. For $n \geqq 4, g(n, n-3)=n$.

Proof. The bipartite graph $\Gamma_{1, n-1}$ is a graph with $n$ vertices, $(n-1)$ edges and path-covering number $(n-2)$. Thus $g(n, n-3) \geqq n$. The reverse inequality is given by Theorem 3.7.

To obtain a lower bound for $g(n, k)$, consider the graph $G=$ $\Gamma_{n-k} \cup \bar{\Gamma}_{k} ;$ then $\mu(G)=k+1$, while $|\mathscr{V}(G)|=n$ and $|\mathscr{E}(G)|=$ $\frac{1}{2}(n-1)(n-k-1)$. This gives:

Proposition 3.14. For $n>k \geqq 1$

$$
g(n, k) \geqq \frac{1}{2}(n-k)(n-k-1)+1 .
$$

The following proposition gives some results that are easily verified:

Proposition 3.15.

(i) $\quad g(n, n)=0, g(n+1, n)=1, g(n+2, n)=2$ for $n \geqq 1$

(ii) $g(6,2)=7$

(iii) $g(n+1, k+1) \geqq g(n, k)$ for $n \geqq k \geqq 1$.

Part (iii) can be seen by letting $G=H \cup\{x\}$ where $H$ is a graph with $n$ vertices, $g(n, k)-1$ edges, and $\mu(H)=k+1$, and $x$ is an isolated vertex with $x \notin \mid$ ith $x \notin \mathscr{V}(H)$. Then $G$ has $(n+1)$ vertices, $g(n, k)-1$ edges, and $(G)=k+2$. 
In the case $k=1$, the upper bound in (3.8) is seen to be the same as the lower bound in (3.15) and hence equality holds for $g(n, k)$ in both inequalities. However, Corollary 3.13 shows that the upper bound in (3.8) and not the lower bound in (3.15) is achieved in the case $k=n-3$. 'Part (ii) of Proposition 3.15 shows a case where the lower bound and not the upper bound is achieved. It is conjectured that for small values of $k, g(n, k)$ is close to the lower bound in (3.15), while for large values of $k, g(n, k)$ is closer to the upper bound in (3.8).

We now turn to another extremal problem. Let $v$ and $n$ be integers with $0 \leqq v \leqq n$. Define:

$h(n, v)=\operatorname{Min}\{k:$ every graph, $G$, with $|\mathscr{V}(G)|=n$ and $\rho(x) \geqq v$

$$
\text { for every } x \in \mathscr{V}(G) \text {, has } \mu(G) \leqq k\} \text {. }
$$

THEOREM 3.16.

$$
(n, v)=\left\{\begin{array}{ccc}
1 & \text { if } & v \geqq \frac{n}{2} \\
n-2 v & \text { if } & v<\frac{n}{2}
\end{array}\right.
$$

Proof. The case $v \geqq \frac{n}{2}$ and the upper bound $h(n, v) \leqq n-2 v$ if $v<\frac{n}{2}$ follows from 0 . Ore's result (the note to Theorem 2.5). If $v<\frac{n}{2}$, let $K=\Gamma_{v, n-v}$. Then clearly $|\mathscr{V}(K)|=n$ and $\rho(x) \geqq v$ for every $x \in \mathscr{V}(G)$; and in [2] (Theorem 2.2.10) we show that $\mu(K)=$ $n-2 v$. Hence

$$
h(n, v) \geqq n-2 v
$$

completing the proof of the theorem.

\section{REFERENCES}

1. C. St. J. A. Nash-Williams, Hamilton arcs and circuits, Recent Trends in Graph Theory, BerlinHeidelberg-New York, Springer-Verlag, (1971), 197-210.

2. S. Noorvash, Covering the vertices of a graph by paths, Hamilton cycles and 1-factors, with related tree enumeration problems, (Ph.D. Thesis, U.C.L.A.) 1972, 8-38.

3. O. Ore, Arc coverings of graphs, Ann. Mat. Ser. IV 55 (1961), 315-321.

4. J. Riordan, An Introduction to Combinatorial Analysis, New York, Wiley, 1958.

Received November 30, 1973. 


\section{CONTENTS}

Zvi Artstein and John A. Burns, Integration of compact set-valued functions

J. A. Beachy and W. D. Blair, Rings whose faithful left ideals are cofaithful

Mark Benard, Characters and Schur indices of the unitary reflection group $[321]^{3}$

H. L. Bentley and B. J. Taylor, Wallman rings ............................. 15

E. Berman, Matrix rings over polynomial identity rings II ............... 37

Simeon M. Berman, A new characterization of characteristic functions of absolutely continuous distributions ........................ 323

Monte B. Boisen, Jr. and Philip B. Sheldon, Pre-Prüfer rings ............ 331

A. K. Boyle and K. R. Goodearl, Rings over which certain modules are injective

J. L. Brenner, R. M. Crabwell and J. Riddell, Covering theorems for finite nonabelian simple groups. $V$

H. H. Brungs, Three questions on duo rings .................................... 345

Iracema M. Bund, Birnbaum-Orlicz spaces of functions on groups ....351

John D. Elwin and Donald R. Short, Branched immersions between 2-manifolds of higher topological type

J. K. Finch, The single valued extension property on a Banach space ................................................................................. 61

J. R. Fisher, A Goldie theorem for differentiably prime rings ............ 71

Eric M. Friedlander, Extension functions for rank 2, torsion free abelian groups ...................................................................... 371

J. Froemke and R. Quackenbusch, The spectrum of an equational class of groupoids

B. J. Gardner, Radicals of supplementary semilattice sums of

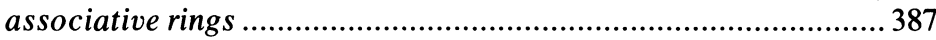

Shmuel Glasner, Relatively invariant measures ...............................393

G. R. Gordh, Jr. and Sibe Mardešić, Characterizing local connectedness in inverse limits...

S. Graf, On the existence of strong liftings in second countable topological spaces

S. Gudder and D. Strawther, Orthogonally additive and orthogonally increasing functions on vector spaces ........................................427

F. Hansen, On one-sided prime ideals .......................................... 79

D. J. Hartfiel and C. J. Maxson, A characterization of the maximal monoids and maximal groups in $\beta x$.

Robert E. Hartwig and S. Brent Morris, The universal flip matrix and the generalized faro-shuffle 


\section{Pacific Journal of Mathematics}

Vol. 58, No. 1

March, 1975

John Allen Beachy and William David Blair, Rings whose faithful left ideals are cofaithful .................................... 1

Herschel Lamar Bentley and Barbara June Taylor, Wallman rings ........ 15

Elizabeth Berman, Matrix rings over polynomial identity rings. II ...... 37

Ann K. Boyle and Kenneth R. Goodearl, Rings over which certain modules are injective ................................. 43

J. L. Brenner, Robert Myrl Cranwell and James Riddell, Covering theorems for finite nonabelian simple groups. $V \ldots \ldots \ldots \ldots \ldots \ldots \ldots \ldots \ldots$

James Kenneth Finch, The single valued extension property on a Banach

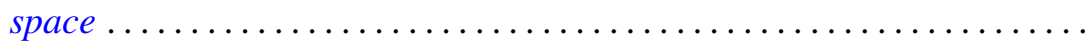

John Robert Fisher, A Goldie theorem for differentiably prime rings........ Friedhelm Hansen, On one-sided prime ideals .................... Jon Craig Helton, Product integrals and the solution of integral equations..........................................

Barry E. Johnson and James Patrick Williams, The range of a normal

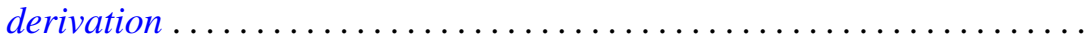

Kurt Kreith, A dynamical criterion for conjugate points ............ 123

Robert Allen McCoy, Baire spaces and hyperspaces .............. 133

John McDonald, Isometries of the disk algebra ................ 143

H. Minc, Doubly stochastic matrices with minimal permanents ......... 155

Shahbaz Noorvash, Covering the vertices of a graph by vertex-disjoint paths. ...

Theodore Windle Palmer, Jordan *-homomorphisms between reduced Banach*-algebras

Donald Steven Passman, On the semisimplicity of group rings of some

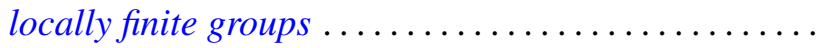

Mario Petrich, Varieties of orthodox bands of groups .

Robert Horace Redfield, The generalized interval topology on distributive

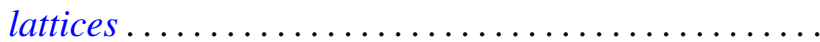

James Wilson Stepp, Algebraic maximal semilattices .... . .

Patrick Noble Stewart, A sheaf theoretic representation of rings with Boolean orthogonalities ........................

Ting-On To and Kai Wing Yip, A generalized Jensen's inequality......... 255

Arnold Lewis Villone, Second order differential operators with self-adjoint

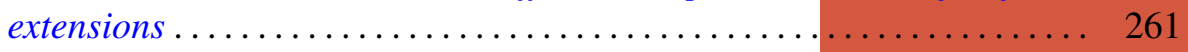

Martin E. Walter, On the structure of the Fourier-Stieltjes algebra ....... 267

John Wermer, Subharmonicity and hulls .................... 283

Edythe Parker Woodruff, A map of $E^{3}$ onto $E^{3}$ taking no disk onto a

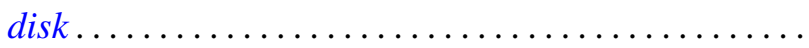

